\title{
FACTORES ASOCIADOS AL NO REPORTE DE EVENTOS ADVERSOS EN SERVICIOS DE APOYO DIAGNÓSTICO 2010-2015
}

\section{FACTORS ASSOCIATED WITH THE NON-REPORTING OF ADVERSE EVENTS IN DIAGNOSTIC SUPPORT SERVICES 2010-2015}

\author{
Luz Mary-Contreras ${ }^{1}$, Camilo-Clavijo², Javier Bula-Romero ${ }^{3}$ \\ Recibido para publicación: Mayo 17 de 2017 - Aprobado para publicación: Junio 28 de 2017
}

\begin{abstract}
RESUMEN
Objetivo: Identificar los factores asociados al no reporte de eventos adversos en los servicios de apoyo diagnóstico en una IPS de primer nivel de atención, con el fin de tomar decisiones orientadas a brindar una atención segura a sus usuarios. Metodología: La presente investigación, corresponde a un estudio descriptivo retrospectivo, de tipo exploratorio. Realizada a partir del análisis de los registros de las actas gestión y seguimiento de los eventos adversos no reportados en el programa de seguridad del paciente de la institución, en el periodo comprendido entre 01 de enero de 2010 a 31 de diciembre de 2015. Resultados: El $46.5 \%$ de los eventos adversos en el servicio de laboratorio clínico y el $45 \%$ de los eventos adversos en el servicio de imágenes diagnósticas durante el período estudiado, no fueron reportados por omisión; situación que afecta la seguridad del paciente indicando una pobre cultura sobre el reporte de los eventos adversos. Conclusiones: El mayor porcentaje de eventos adversos no reportados en los servicios de apoyo diagnóstico durante el período estudiado. Esta situación se atribuyó a que el personal implicado temía que se tomaran acciones punitivas como consecuencia a la presentación de estos sucesos. Recomendaciones: Es importante llevar a cabo un proceso de monitoreo y revisión permanente de los riesgos identificados en las áreas y dependencias de los servicios de apoyo diagnóstico de la institución prestadora de servicios de salud.
\end{abstract}

Palabras clave: Evento, adverso, servicios, diagnóstico (MeSH).

\begin{abstract}
Objective: To identify the factors associated with the non-reporting of adverse events in diagnostic support services in a primary level of care, in order to make decisions aimed at providing a safe care to its users. Methodology: This research corresponds to a retrospective descriptive, exploratory study. Based on the analysis of the records of the management and follow-up records of adverse events not reported in the institution's patient safety program, during the period from January 1, 2010 to December 31, 2015. Results: $46.5 \%$ of adverse events in the clinical laboratory service and $45 \%$ of adverse events in the diagnostic imaging service during the study period were not reported by default; situation that affects the safety of the patient indicating a poor culture on the report of adverse events. Conclusions: The highest percentage of unreported adverse events in diagnostic support services during the study period was not carried out of fear, this situation was attributed to the staff involved fearing that punitive actions were taken as a consequence of the presentation of these events. Recommendations: It is important to carry out a process of permanent monitoring and review of the risks identified in the areas and dependencies of the diagnostic support services of the institution providing health services.
\end{abstract}

Keywords: Adverse, event, diagnostic, services (MeSH).

\footnotetext{
${ }^{1}$ Enfermera Especialista Gerencia de la calidad en salud

${ }^{2}$ M.Sc en Enfermería con énfasis en Gerencia de servicios de salud

${ }^{3}$ Enfermero M.Sc \& PhD (c) en Enfermería. Profesor, Facultad Ciencias de la Salud, Universidad de Córdoba, Montería, Colombia.

Correspondencia javierbula@correo.unicordoba.edu.co
} 


\section{INTRODUCCIÓN}

En los últimos años la calidad y la seguridad se han convertido en una preocupación creciente y una prioridad de los sistemas sanitarios (1). El no reporte de los eventos adversos de cualquier dependencia o área donde se preste una atención en salud no solo compromete la seguridad de los pacientes, sino también los procesos de calidad asociados a la prestación de los servicios de una institución.

La Organización Mundial de la Salud (OMS 2008) estima que cada año a escala mundial, millones de pacientes presentan Eventos Adversos (E.A) por causa de una atención insegura sin importar el desarrollo socioeconómico de los países (2).

Colombia no es ajena a esta realidad, es por ello que a nivel nacional el Ministerio de la Protección Social, viene impulsando una política de seguridad del paciente, liderada por el Sistema Obligatorio de Garantía de la Calidad de la Atención en Salud, cuyo objetivo es prevenir la ocurrencia de situaciones que afectan la seguridad del paciente, reducir y analizar la ocurrencia de Eventos Adversos para contar con instituciones seguras y competitivas (3).

Esta política de calidad se enfoca en la seguridad del paciente y de los profesionales de salud, su proceso de implementación dentro de las instituciones, se lleva a cabo por medio de un programa de seguridad del paciente que permite identificar los riesgos inherentes a la atención y gestionar los eventos presentados, sin embargo; la existencia de un sistema de reporte de E.A no garantiza que el personal de salud lo utilice a pesar de ser esta una actividad de obligatorio cumplimiento.

En la institución objeto de estudio, a pesar de existir una política y un programa de seguridad del paciente socializado a todo el personal, se evidencia que hay una tendencia al no reporte de Eventis Adversos (EA) en los servicios de apoyo diagnóstico, situación que dificulta el proceso de gestión del riesgo y los procesos de calidad asociados a una atención segura en los servicios de salud.

Acorde con lo planteado, la presente investigación se planteó como objetivo general identificar los factores asociados al no reporte de eventos adversos en los servicios de apoyo diagnóstico en una Institución Prestadora de servicios de Salud (I.P.S) de primer nivel de atención, con el fin de tomar decisiones orientadas a brindar una atención segura a sus usuarios que adicionalmente garantice la confianza y reconocimiento del servicio en el sector salud.

\section{METODOLOGÍA}

La presente investigación, corresponde a un estudio descriptivo, retrospectivo, de tipo exploratorio. Realizada a partir del análisis de los registros de las actas gestión y seguimiento de los eventos adversos no reportados en el programa de seguridad del paciente de la institución, en el periodo comprendido entre 01 de enero de 2010 a 31 de diciembre de 2015. Para caracterizar los eventos adversos no reportados en los servicios de apoyo diagnóstico de la institución, se realizó un análisis descriptivo retrospectivo del contenido de las actas de gestión de los eventos adversos y un análisis de las quejas y reclamos asociados a la presencia de éstos en los servicios en mención.

\section{Unidad de Análisis}

La unidad de análisis estuvo conformada por las actas de seguimiento y gestión del evento adverso y los formatos de quejas, peticiones y reclamos presentados por los usuarios después de la ocurrencia de los eventos adversos o incidentes presentados durante la atención en salud en los servicios de apoyo diagnóstico de la institución objeto de estudio. 


\section{Muestra}

La muestra estuvo constituida por el número de eventos adversos no reportados en los servicios de apoyo diagnóstico en el periodo 2010- 2015 ( $n=110$ E.A No reportados).

\section{Fase retrospectiva del estudio}

En esta fase se tomó la información de los eventos adversos no reportados en los servicios de apoyo diagnóstico de la institución, a partir de los registros oficiales de las actas de seguimiento y gestión del evento adverso. Esta información se registró en una base de datos en Excel para facilitar el manejo de la misma.

Estas actas permitieron identificar los eventos adversos que no fueron reportados a través del programa de seguridad del paciente. Las fuentes de información utilizadas para la construcción de la evidencia fueron las quejas, peticiones, y reclamos presentados por los usuarios después de la ocurrencia de los eventos adversos o incidentes de la atención en salud. Estas actas permitieron extraer información sobre las personas implicadas en la situación de no reporte, los factores asociados al no reporte y el análisis causal de la ocurrencia del evento adverso y del no reporte del mismo, convirtiéndose en el insumo de esta investigación.

A partir de los hallazgos documentados en las actas se realizaron análisis univariados, los cuales permitieron describir y sintetizar los datos de una muestra conocida, a través de la estadística básica. En este proceso se obtuvieron los datos sociodemográficos y descriptivos del talento humano implicado en el no reporte de eventos adversos, permitiendo así caracterizarlos según porcentajes y medidas de tendencia central.

\section{Criterios de Inclusión}

Para la selección de las actas de seguimiento y gestión de los eventos adversos asociados a la atención de los servicios de apoyo diagnóstico de la institución objeto de estudio, se tuvieron en cuenta los siguientes criterios de inclusión:

- Actas de gestión y seguimiento de los eventos adversos no reportados e identificados en el periodo de tiempo del estudio.

- Actasquecumplieronconcriteriosdecalidad en sus registros, tales como: descripción del evento adverso no reportado, servicio en el que ocurrió el evento adverso, personal que estuvo implicado en el no reporte del evento adverso.

\section{Criterios de Exclusión}

Registros de actas de gestión y seguimiento del evento adverso con información incompleta $u$ omisión de la descripción del evento adverso no reportado.

\section{Descripción del proceso operativo para la recolección y sistematización de la información.}

Para dar respuesta a la pregunta de investigación y llevar a cabo el cumplimiento de los objetivos del estudio, el primer paso que se realizó fue interactuar con las directivas de la institución objeto de estudio con el fin de dar a conocer la propuesta de investigación, los objetivos del estudio y de esta forma obtener el aval para llevar a cabo el proyecto de investigación.

Posterior a este primer acercamiento, se obtuvo el aval de la IPS para recolectar la información necesaria como se describe a continuación:

Se aplicó una lista de chequeo para verificar la implementación del programa de seguridad del paciente. Esta lista de chequeo permitió conocer el porcentaje de cumplimiento de los criterios sobre los cuales se implementó el programa de seguridad del paciente, identificando las fortalezas, debilidades y fallas que se presentaron en el proceso de implementación del mismo. 
Se seleccionaron las actas de seguimiento de gestión de reporte de eventos adversos, que cumplieron con los criterios de inclusión, con el fin de conocer el número de casos ocurridos, tipo de evento adverso presentado, fuente de obtención de la información, dependencia e implicados en la ocurrencia de eventos adversos no reportados y finalmente, los factores asociados al no reporte de los mismos en el período de tiempo señalado. A cada una de las actas se le asignó un código numérico que permitió su identificación para mantener la confidencialidad de los datos y la información de los sujetos y eventos adversos que sucedieron en los servicios de apoyo diagnóstico.

Por último, por medio del análisis de la información de las actas, se identificó el talento humano implicado en las situaciones de no reporte de eventos adversos, a través de la aplicación de la ficha de caracterización del talento humano, luego se recurrió al análisis de las hojas de vida del personal implicado con el fin de identificar y describir las características del talento humano asociado al no reporte de eventos adversos en los servicios de apoyo diagnóstico de la IPS objeto de estudio. Las características del talento humano que fueron analizadas corresponden a la edad, escolaridad, tipo de vinculación, tiempo de vinculación y tiempo de experiencia en el área de desempeño.

Para dar respuesta a los objetivos específicos se dispuso de los instrumentos señalados en el apartado anterior, estableciendo los análisis correspondientes a los hallazgos documentados.

El análisis y la discusión de los resultados se hizo utilizando la literatura disponible sobre el fenómeno de estudio, con el fin de encontrar puntos de convergencia o divergencia de acuerdo a los reportes encontrados.

\section{Consideraciones éticas}

Para la realización de la investigación no se requirió la utilización de consentimiento informado, dado que la información fue recolectada desde fuentes documentales. Se tuvo en cuenta la confidencialidad de la información obtenida, sobre todo la relacionada con el talento humano implicado en las situaciones de no reporte, aclarando que no se afectarían las condiciones laborales del mismo.

Se garantizó la confidencialidad de los datos, asignando aleatoriamente códigos numéricos a cada una de las actas revisadas, estos códigos se exportaron a la matriz operativa en la que se consignaron las variables de interés.

Los datos recolectados fueron transcritos fielmente a la base de datos en la cual se condensó el acopio de la información, siendo verificada para identificar duplicidad de los datos o errores en la transcripción de los mismos. Por último la información no fue alterada ni sustituida en ningún momento del proceso, garantizando la veracidad de la información para el desarrollo del proyecto investigativo.

La información obtenida en el acopio de los datos se utilizó con fines académicos, la socialización de los resultados tiene como objetivo mejorar los procesos operativos del programa de seguridad del paciente y la cultura organizacional para el reporte de los eventos adversos al interior de la institución.

En cuanto a la gestión documental de las actas de gestión de los eventos adversos, se garantizó la fidelidad de la información de los registros consignados, sin alterar, sustituir o remplazar el contenido de las mismas.

\section{RESULTADOS}

El comportamiento de los eventos adversos no reportados en la institución objeto de estudio, muestra que el mayor número de casos no reportados en el servicio de laboratorio clínico, ocurrió durante el año 2010, (19 casos); mientras 
que para el área de imágenes diagnósticas el mayor número de casos se presentó en el 2010 ( 7 casos), manteniendo una tendencia de 5 casos por año para el 2011 y 2012, con una disminución significativa del número de casos reportados durante el último año estudiado (1 caso identificado en 2015).Ver figura 1.

\section{Eventos Adversos No Reportados 2010 - 2015} La edad promedio del talento humano implicado en el proceso de no reporte de los EA osciló entre los 23 y 56 años, con una media de 35 años y una moda de 32 años. El $50 \%$ de ellos se encontraba en nómina y el otro $50 \%$ se encontraba vinculado por prestación de servicios. En cuanto al nivel de formación se puede apreciar que el 37,5\% del recurso humano tenía formación tecnológica, el $50 \%$ formación profesional y el $25 \%$ restante formación técnica, reportado un tiempo de experiencia que oscilaba entre los 5 a 10 años.

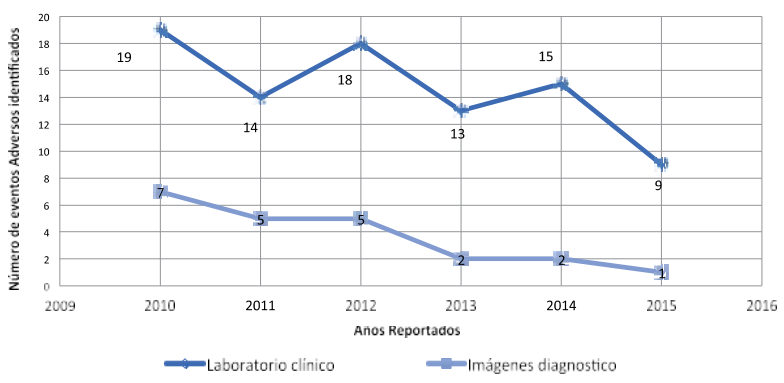

Figura 1. Eventos adversos no reportados en la IPS de primer nivel $2010-2015$.

Fuente: Actas de gestión de los E.A no reportados en el servicio de laboratorio, años 2010-2015 en la IPS objeto de estudio.

El mayor porcentaje de eventos adversos no reportados en el servicio de laboratorio clínico ocurrió durante la etapa pre analítica (56\%), y el porcentaje restante (40\%) se presentó en la etapa pos analítica del proceso Figura 2.

\section{Eventos adversos no reportados en el servicio de laboratorio clínico}

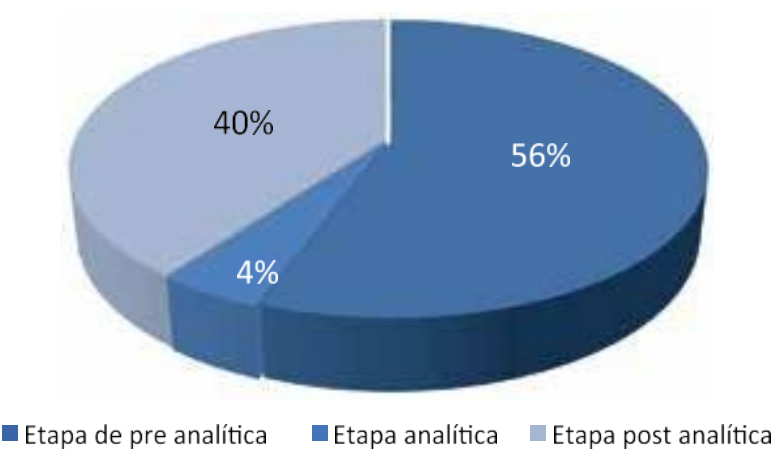

Figura 2. Porcentaje de eventos adversos no reportados en el servicio de laboratorio clínico según la etapa del proceso años 2010-2015.

Fuente: Actas de gestión de los E.A no reportados en el servicio de laboratorio, años 2010-2015 en la IPS objeto de estudio.

Al discriminar las distribuciones porcentuales de los eventos adversos no reportados en el servicio de laboratorio clínico se puede apreciar en la tabla 1 , que el $18.2 \%$ de los eventos adversos identificados correspondían a retrasos en los resultados, el $15.9 \%$ se atribuía a errores en las condiciones de la muestra y el $14.8 \%$ a errores relacionados con los resultados. En menor proporción se presentaron errores en la identificación del paciente (13.6\%), seguido de la identificación errónea de las muestras con un $11.4 \%$.

Tabla 1. Porcentaje de E.A no reportados servicio de laboratorio clínico años 2010-2015.

\section{E.A no reportados identificados}

Distribución

\begin{tabular}{ll}
\hline Retrasos en los resultados & $18.2 \%$ \\
Errores en las condiciones de la muestra & $15.9 \%$ \\
Errores relacionados con los resultados & $14.8 \%$
\end{tabular}

Fuente: Actas de gestión de los E.A no reportados en el servicio de laboratorio, años 2010-2015 en la IPS objeto de estudio $E$. 
Al determinar los factores asociados al no reporte de eventos adversos en el servicio de laboratorio clínico, se puedo identificar que el $46.5 \%$ no consideró importante reportar la situación presentada, el $29.5 \%$ omitió el reporte, y el $23.86 \%$ no lo reportó por miedo. Figura 3.

Factores asociados al no reporte de E.A en el servicio de laboratorio clínico

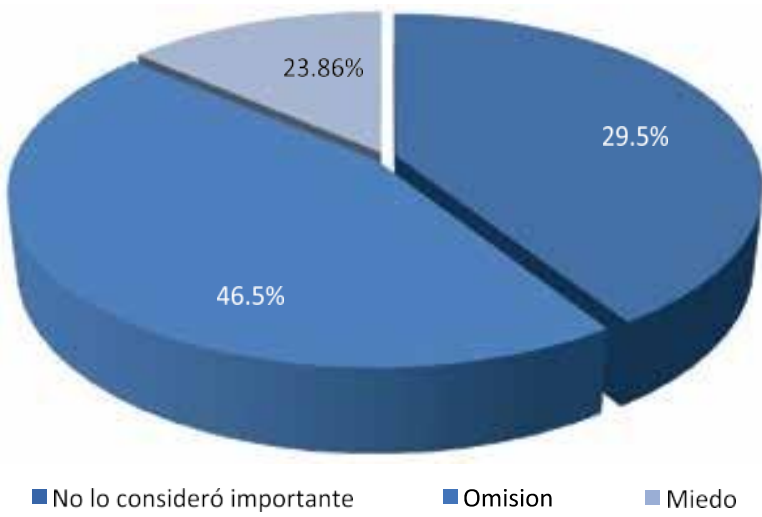

Figura 3. Factores asociados al no reporte de E.A en el servicio de laboratorio clínico de una IPS de primer nivel de atención.

Fuente: Actas de gestión de los E.A no reportados en el servicio de laboratorio, años 2010-2015 en la IPS objeto de estudio.

Para el área de radiología e imágenes diagnósticas la mayor distribución porcentual de eventos adversos no reportados se presentó por a retrasos en los resultados con el $22.7 \%$, mientras el $18.2 \%$ se presentó debido a errores en la identificación del paciente. El $45 \%$ de las causas de no reporte de eventos adversos en el servicio de imágenes diagnósticas corresponde a omisión, el $41 \%$ no se reportaron por que no fueron considerados importantes y el $14 \%$ de los eventos adversos identificados no fueron reportados por miedo a las implicaciones del reporte, como se evidencia en la figura 4.

\section{Factores asociados al no reporte de eventos adversos en el servicio de apoyo diagnóstico}

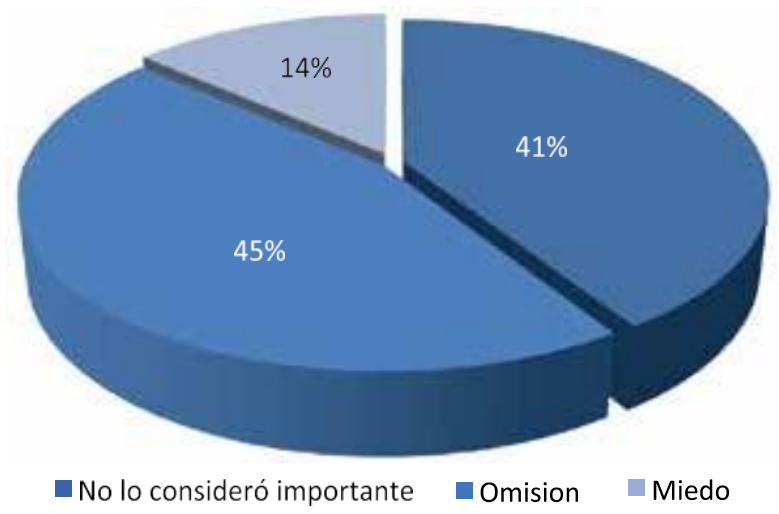

Figura 4. Factores asociados al no reporte de E.A en el servicio de apoyo diagnóstico de una I.P.S de primer nivel de atención. Año 2010-2015

Fuente: Actas de gestión de los E.A no reportados en los servicios de laboratorio clínico y toma de muestras, años 2010-2015 en la IPS objeto de estudio.

\section{DISCUSIÓN}

La literatura disponible sobre la gestión de los eventos adversos, evidencia que un porcentaje considerable de los mismos no son reportados cuando existe una equivocación y esta no afecta la condición del paciente. $(3,4,5)$ En los hallazgos de este estudio se puede identificar que el número de eventos adversos no reportados en los servicios de apoyo diagnóstico en los períodos de estudio corresponden a $n=110$ eventos, que involucran no solo al talento humano que participa en la atención directa e indirecta del paciente, sino también los procesos de calidad de las áreas donde se desarrolla la prestación del servicio.

Cuando se hace un análisis del número de eventos adversos no reportados, discriminando las áreas de ocurrencia, se puede observar que en el área de laboratorio clínico se presenta el mayor número de casos identificados, los retrasos en el resultado ocupan el $18.2 \%$ de los eventos no reportados, seguido de errores asociados al diagnóstico con un $15.9 \%$ y en 
menor proporción eventos adversos asociados a fallas en el sistema y fallas en los equipos biomédicos con un $4.5 \%$.

Al describir el análisis de los eventos adversos en el servicio de laboratorio clínico de acuerdo a las fases del proceso, se muestra que en la etapa pre analítica ocurren el mayor número de eventos adversos no reportados. Los hallazgos de este estudio indican que el $56 \%$ del total de los eventos adversos no reportados que fueron identificados, corresponden a esta fase del proceso de análisis.

Estos resultados coinciden con estudios como el de Barba 2014 (6) quien indica que es en esta etapa donde se produce el mayor número de errores reportados y por lo tanto, la principal fuente de eventos adversos relacionados con el paciente y su muestra biológica. Según un estudio realizado por la Sociedad Española de Bioquímica Clínica y Patología Molecular

(7) el $88.8 \%$ de los errores producidos en la etapa pre analítica se relaciona con rechazo de las muestras clínicas, y estas corresponden a muestras no recibidas por estar hemolizadas, coaguladas o ser insuficientes. Otros de los aspectos contemplados dentro de la etapa pre analítica indican que es en esta fase en la que el mayor número de colaboradores (profesionales y técnicos) se ven involucrados, tales como los médicos solicitantes, las auxiliares de laboratorio, bacteriólogos, el personal administrativo y el propio paciente.

En la etapa pos analítica el porcentaje de eventos adversos para este estudio fue del $40 \%$ y estos estuvieron asociados con retrasos en los resultados (8). Mientras que en la etapa analítica el porcentaje reportado fue del $4 \%$, en esta etapa existe la menor ocurrencia de errores ya que se encuentra sujeta a controles de calidad tanto interno como externo, sin embargo los errores que aparecen están relacionados con fallas en el sistema y los equipos biomédicos (6).
En términos generales puede decirse que los eventos adversos en un laboratorio clínico pueden atribuirse a deficiencias en la planificación, organización, funcionamiento integral del servicio, fallas en los equipos de medición y sobre todo fallas en la implementación de procedimientos operativos en cada una de las etapas del proceso analítico. Sin embargo es claro que en la fase pre analítica es donde ocurre el mayor porcentaje de errores identificados $(9,10,11,12,13)$.

Unestudio realizado por Plebani \& Carraro 2007 (14) detectó que de 4.940 pruebas realizadas en un servicio de laboratorio clínico se detectaron 189 eventos adversos, de los cuales el $68.2 \%$ pertenecían a la fase pre analítica, $13.3 \%$ a la fase analítica y $18.5 \%$ a la fase pos analítica. Estos autores indican que el $74 \%$ no tuvo ningún efecto en los pacientes pero el $19.6 \%$ ocasionó la solicitud de nuevos estudios y el $6.4 \%$ afectó la terapia del paciente. A diferencia de estos hallazgos, nuestro estudio no reportó que algún efecto adverso generara modificación en el manejo y pronóstico del paciente, lo cual fue una limitación para el estudio puesto que las actas de gestión y seguimiento de los eventos adversos no logra describir aspectos relacionados con lo anteriormente expuesto. Carraro P et al, 2007.

En relación al servicio de imágenes diagnósticas, diversas publicaciones indican que en promedio el error de un radiólogo alcanza entre $20-30 \%$ de los informes, estos errores están asociados al proceso diagnóstico y corresponde a aquellos casos en el que el radiólogo no ve o no informa los hallazgos de la imagen (15). Los hallazgos de este estudio muestran en términos generales que los errores del proceso diagnóstico representaron el 5\% de los eventos adversos no reportados, mientras que el $95.5 \%$ estuvo asociado a eventos adversos propios de errores de logística que incluyen retraso en los resultados con $22.7 \%$, errores en la identificación $18.2 \%$ y extravío de la placa $9.1 \%$. 
Cuando se examinan los factores asociados al no reporte de eventos adversos se puede identificar que el mayor porcentaje $46.5 \%$ se atribuye a que los sujetos implicados no lo consideran importante, el $29.5 \%$ omitió el reporte y el $23 \%$ no reportó por miedo. Algunos autores como Helmreich, 2011 (16) indican que el éxito de un sistema de reporte está determinado por las actitudes y percepciones de los profesionales y los técnicos que están al frente del cuidado de la salud de las personas. Este autor menciona que las causas del no reporte pueden deberse al no considerar importante reportarlo, y además considerar que el reporte pudiera generar algún cambio, no hubo suficiente tiempo para reportarlo, no querer que algo negativo pasara a los colaboradores implicados en el evento, y no querer dejar expuesto el nombre de la persona implicada.

Otros autores como Vincent C et. al. 1999 (18) indican que en el proceso de entender por qué no se reportaban los EA se encontró, en orden de importancia: "por parecerle innecesario al personal", por el "incremento de la carga de trabajo", por "miedo al castigo", por "olvido" y finalmente por la "probabilidad de un litigio".

De acuerdo con la guía de buenas prácticas del Ministerio de Salud (17) para la implementación del programa de seguridad del paciente, la Gerencia debe asignar un presupuesto económico para las actividades de seguridad, se debe conformar un equipo líder de seguridad, se da entrenamiento a los líderes en los aspectos de seguridad del paciente, se capacita continuamente a los colaboradores en las prácticas seguras recomendadas y se implementan las acciones de mejora (17). La gestión del riesgo es un proceso fundamental por medio del cual se identifica y aprovecha oportunidades para mejorar el desempeño y emprender acciones para evitar o reducir las oportunidades de que algo salga mal. MPS, 2010 (17)
La implementación del programa de seguridad del paciente en la IPS objeto de estudio no es garante del éxito del proceso de reporte deE.A, y uno de los factores que se puede apreciar al respecto es que el personal atemorizado por las retaliaciones subregistran y en ocasiones hasta oculta los eventos adversos evitando ser señalados o culpables por los jefes de área o por sus mismos compañeros $(19,20,21)$.

Este planteamiento concuerda con el estudio realizado por Gaitán 2009 (22), en cuatro hospitales de Colombia, donde se observó que la resistencia del personal de salud para reportar un evento adverso surgía de "considerar el proceso como una auditoria que puede traer consecuencias negativas".

Es importante indicar que cada punto del proceso de cuidado de los pacientes contiene cierto grado de riesgo inherente; que hacen que en la práctica sea imposible la desaparición total de posibles eventos adversos, sin embargo, esta realidad no tiene que ser la causa de que no se actúe sobre aquellos efectos que puedan ser prevenibles y por tanto evitables $(23,24,25,26)$.

Finalmente, entender que el proceso de reporte de los eventos adversos en una institución va de la mano con la cultura del reporte que la institución fomente entre sus colaboradores. Así mismo, desde el área de gestión y calidad se hace necesario fortalecer el programa de seguridad del paciente involucrando no solo al personal asistencial sino también a los usuarios y sus familias como parte activa de los procesos de calidad de la institución. (30, 31, 32, 33, 34, 35).

\section{CONCLUSIONES}

Una de las razones relevantes del no reporte de eventos adversos en el servicio de laboratorio clínico de la IPS objeto de estudio hace referencia a que el talento humano implicado en este proceso no consideró importante hacer el reporte, si éste no afectaba la condición del 
paciente. Hecho que evidencia la necesidad de implementar estrategias de educación en el ámbito de la seguridad del paciente y específicamente en el reporte oportuno de eventos adversos.

El mayor porcentaje de los eventos adversos en el servicio de laboratorio clínico (46.5\%) y el mayor porcentaje de los eventos adversos en el servicio de imágenes diagnósticas (45\%) durante el período estudiado, no fueron reportados por omisión; situación que afecta la seguridad del paciente indicando una pobre cultura sobre el reporte de los eventos adversos.

El mayor porcentaje de eventos adversos no reportados en los servicios de apoyo diagnóstico durante el período estudiado no se realizó por miedo, esta situación se atribuyó a que el personal implicado temía que se tomaran acciones punitivas como consecuencia a la presentación de estos sucesos.

El mayor porcentaje de eventos adversos no reportados en el laboratorio clínico (56\%) correspondieron a la fase pre analítica y el (95.5\%) de los eventos adversos no reportados en el servicio de imágenes diagnósticas, fueron atribuidos a errores de logística.

\section{RECOMENDACIONES}

Es importante llevar a cabo un proceso de monitoreo y revisión permanente de los riesgos identificados en las áreas y dependencias de los servicios de apoyo diagnóstico de la IPS, junto con el apoyo de la alta gerencia con el fin de asegurar que las acciones tomadas sean implementadas para beneficio y seguridad de los usuarios que solicitan los servicios.

Realizar búsqueda activa de eventos adversos por medio de monitoreo y seguimiento de las peticiones, quejas, reclamos y sugerencias (PQRS) y realizar los análisis retrospectivos como estrategias de apoyo en la identificación de EA.
Se debe adoptar un sistema de gestión de riesgo por medio del cual se puedan analizar los diferentes procesos de las áreas de los servicios de apoyo diagnóstico de la institución a través del programa de seguridad del paciente con el fin de mitigar posibles fallas en los procesos de atención.

Desde el área de gestión humana es importante trabajar directamente con los colaboradores de la IPS el reporte de los eventos adversos, a través de acciones educativas que permitan la sensibilización y consientización en la cultura del reporte de los mismos para fortalecer la seguridad del paciente y brindar una atención de calidad.

\section{CONFLICTO DE INTERÉS}

Los autores declaran no tener conflicto de interés.

\section{REFERENCIAS}

1. Pascual Parrilla Paricio, J. I. Landa García, AEC Asociación Española de Cirujanos. Disponible EN: http://www. medicapanamericana.com/Libros/ Libro/3771/Cirugia-AEC.html. Consultado 05 de septiembre de 2015.

2. Organización Mundial de la Salud. Alianza Mundial para la Seguridad del Paciente: la investigación en seguridad del paciente: la investigación en seguridad del paciente, mayor conocimiento para la atención más segura. Francia: OMS. 2008 p 11.

3. Ministerio de la Protección Social. Lineamientos para la implementación de la política de seguridad del paciente. Bogotá D.C. Noviembre 2008 p 7.

4. Ministerio de la Protección Social. El Estudio IBEAS y la Política de Seguridad del Paciente en Colombia. Bogotá 2010. [Sitio en internet].disponible en http://www. 
minproteccionsocial.gov.co/VBeContent/ Library/Documents/ DocNewsNo14913. pdf.

5. Kerguelen, C. ¿Qué implica hablar de una cultura de seguridad de pacientes? En: Centro de gestión hospitalaria. Vía Salud. 2008. No 41. P. 4-10.

6. Barba J. Contribución del laboratorio clínico en la seguridad del paciente. Revista Latinoamericana Patología Clínica Médica Lab. 2014; 61 (1): 11-23.

7. Barba J. Contribución del laboratorio clínico en la seguridad del paciente. Revista Latinoamericana Patología Clínica Médica Lab. 2014; 61 (1): 11-23.

8. Barba J. Contribución del laboratorio clínico en la seguridad del paciente. Revista Latinoamericana Patología Clínica Médica Lab. 2014; 61 (1): 11-23.

9. Almazán S, Cercenado S, Iglesias F. Área de Seguridad del Paciente y Calidad Percibida del SESCAM. Plan estratégico de seguridad del paciente. Servicio de Salud de Castilla la Mancha. 2009-2012.

10. Angüiano, et al. Errores en el laboratorio clínico; evaluación de tipos y frecuencias. Elsevier Medicina Universitaria, 13(52):133-138. México 2011.

11. Aranaz, J. et al. Gestión Sanitaria. Calidad y Seguridad de los Pacientes. Fundación Mapfre. España. 2008.

12. Burbano et al. Causas del no reporte de eventos adversos en una Institución Prestadora de Servicios de Salud en Pasto. Revista universidad y salud, Vol. 15(2) Págs. 187 - 195. Año 2013 - Nariño, Colombia.

13. Evans SM, Berry JG, Smith BJ, Esterman A, Selim $P$, Shaughnessy $J O$ et al. Attitudes and barriers to incident reporting: a collaborative hospital study. Qual Saf Health Care. 2006; 15:39-43.

14. Plebani M \& Carraro, Errors in a Stat Laboratory: Types and Frequencies 10 Years Later. Clinical Chemistry. 2007; 53 (7):1338-1342.

15. García C. Anatomía del error en radiología. Revista chilena de Radiología, Vol. $9 \mathrm{~N}^{\circ} 3$. 2003 p.144-150.

16. Helmreich R. Identifying Barriers to the Success of a Reporting System. Agency for Healthcare Research and Quality (US) [Internet]. [Cited 26 April 2016]; Available from: http://www.ncbi.nlm.nih.gov/books/ NBK20544/\#A4544.

17. Vincent C, Stanhope N, and Crowley M. Reasons for not reporting adverse incidents: an empirical study. J Eval Clin Pract. 1999; 5(1):13-21.

18. Ministerio de la Protección Social. Norma técnica: Buenas prácticas para la seguridad del paciente en la atención en salud. Unidad Sectorial de Normalización en salud. Dirección General de Calidad de Servicios. Bogotá, D.C.: El Ministerio, 2009.

19. Ministerio de la Protección Social. El Estudio IBEAS y la Política de Seguridad del Paciente en Colombia. Bogotá 2010. [Sitio en internet].disponible en http://www. minproteccionsocial.gov.co/VBeContent/ Library/Documents/ DocNewsNo14913. pdf.

20. Farley DO, Haviland A Champagne $S$ Jain AK, Battles JB, Munier WB. Adverse event reporting practices by US hospitals: results of a national survey. Qual Saf Health Care. 2008; 17:416-23. 
21. Gaitán H, Gómez P, Eslava J. Actitudes del personal en la vigilancia de eventos adversos intrahospitalarios en Colombia. Rev. Salud Pública. 2009; 11(5):745-753.

22. López D. Et al. Seguridad del paciente en la prestación de servicios de salud de primer nivel de atención. Monografía para optar al título de especialista en auditoria en salud. Universidad de Antioquia Facultad de Salud Pública, Medellín 2008.

23. Mendoza, J. Sobre la Definición de la Salud, Disponible en http://encolombia. com/medicina/revistas-medicas/ academedicina/va-52/m-03jmendoza/ Consultado 15 de Agosto de 2015.

24. Ministerio de la Protección Social. Lineamientos para la implementación de la política de seguridad del paciente. Bogotá D.C. Noviembre 2008.

25. Ministerio de Salud. Observatorio de Calidad de la atención en salud. INCAS, Colombia, 2009.

26. Ministerio de Sanidad y Consumo, Universidad Miguel Hernández. Estudio Nacional sobre los Efectos Adversos ligados a la hospitalización ENEAS. Madrid 2006. [Sitio en internet]. http://www.msc. es/organización/sns/plancalidadSNS/pdf/ excelencia/opscsp2.pdf Consultado: 08 de agosto de 2015.

27. Ministerio de Salud de Chile. Estándares mínimos de acreditación para los prestadores institucionales: estándares generales para establecimientos de imagenología. Chile 2007. Disponible en: http://www.minsal.cl/ici/destacados/ Estandares_Imagenologia_definitiv o_WEB. pdf Consultado el 08 de agosto de 2015.

28. Ministerio de la Protección Social. El
Estudio IBEAS y la Política de Seguridad del Paciente en Colombia. Bogotá 2010. [Sitio en internet]. URL disponible en http://www. minproteccionsocial.gov.co/VBeConte nt/ Library/Documents/ DocNewsNo14913. pdf Consultado: 08 de agosto de 2015.

29. Ministerio de la Protección Social. El estudio IBEAS y la política de seguridad del paciente en Colombia. Dirección General de Calidad de Servicios. Bogotá D.C: El Ministerio.2011.

30. Ministerio de la Protección Social. Norma técnica: Buenas prácticas para la seguridad del paciente en la atención en salud. Unidad Sectorial de Normalización en salud. Dirección General de Calidad de Servicios. Bogotá, D.C.: El Ministerio, 2009.

31. Ministerio de la Protección Social, Atención de Salud del Sistema General de Seguridad Social en Salud. Colombia, Abril 3 de 2006.

32. OPS-FEPPEN. Calidad de los Servicios de Salud en América Latina y el Caribe: Desafíos para la Enfermería. Programa de Organización y Gestión de Sistemas y Servicios de Salud (HSO). 2001.

33. Organización Mundial de la Salud. Alianza Mundial para la Seguridad del Paciente: la investigación en seguridad del paciente, mayor conocimiento para la atención más segura. Francia: OMS. 2008. $p 11$.

34. Ortega D. et al. Prevención de Riesgo en radiología: el error y el radiólogo. Revista Chilena de Radiología. Vol. 8 № 3, año 2002. Chile 2002. 